\title{
A Three-Stage Procedure for Controlled Islanding to Prevent Wide-Area Blackouts
}

\author{
Hongbo Shao ${ }^{1}$, Yubin Mao ${ }^{1}$, Yongmin Liu ${ }^{1}$, Wanxun Liu ${ }^{1}$, Sipei Sun ${ }^{1}$, Peng Jia ${ }^{1}$, \\ Fufeng Miao ${ }^{1}$, Li Yang ${ }^{2}{ }^{*}$, Chang Han ${ }^{2}$ and Bo Zhang ${ }^{3}$ \\ 1 State Grid Henan Economic Research Institute, Zhengzhou 450000, China; hongbo_shao@sina.com (H.S.); \\ yubin_mao@sina.com (Y.M.); liuamin@163.com (Y.L.); wanxun_liu@sina.com (W.L.); \\ sunsipei@gmail.com (S.S.); tjujp123@126.com (P.J.); miaofufeng@mail.iee.ac.cn (F.M.) \\ 2 School of Electrical Engineering, Zhejiang University, Hangzhou 310027, China; changhanzju@163.com \\ 3 Department of Electrical, Computer and Systems Engineering, Rensselaer Polytechnic Institute, Troy, \\ NY 12180, USA; bozhang321@gmail.com \\ * Correspondence: eeyangli@zju.edu.cn; Tel.: +86-571-8795-1542
}

Received: 11 October 2018; Accepted: 4 November 2018; Published: 7 November 2018

\begin{abstract}
Controlled islanding has been proposed as a last resort action to stop blackouts from happening when all standard methods have failed. Successful controlled islanding has to deal with three important issues: when, and where to island, and the evaluation of the dynamic stability in each island after islanding. This paper provides a framework for preventing wide-area blackouts using wide area measurement systems (WAMS), which consists of three stages to execute a successful islanding strategy. Normally, power system collapses and blackouts occur shortly after a cascading outage stage. Using such circumstances, an adapted single machine equivalent (SIME) method was used online to determine transient stability before blackout was imminent, and was then employed to determine when to island based on transient instability. In addition, SIME was adopted to assess the dynamic stability in each island after islanding, and to confirm that the chosen candidate island cutsets were stable before controlled islanding was undertaken. To decide where to island, all possible islanding cutsets were provided using the power flow (PF) tracing method. SIME helped to find the best candidate islanding cutset with the minimal PF imbalance, which is also a transiently stable islanding strategy. In case no possible island cutset existed, corresponding corrective actions such as load shedding and critical generator tripping, were performed in each formed island. Finally, an IEEE 39-bus power system with 10 units was employed to test this framework for a three-stage controlled islanding strategy to prevent imminent blackouts.
\end{abstract}

Keywords: blackouts; cascading outages; controlled islanding; power flow (PF) tracing; slow coherency

\section{Introduction}

Power blackouts are still a recurring problem around the world and the search continues to develop ever-better methods for their prediction and prevention. This paper concentrates on controlled islanding, which has been proposed as the last-line of defense when all the usual protection measures have failed and a blackout is imminent [1,2].

Usually, as is the case in the US/Canada [3], the dynamic process accompanying a blackout consists of four stages [4]: slow cascading trips, fast cascading, oscillation and collapse. In the slow cascading stage, transmission lines are tripped by protection due to overloads. Once a line is tripped, the power flow $(\mathrm{PF})$ on the tripped line has to be shared by neighboring lines, which may cause violation of the thermal rating on adjacent lines and lead to further cascading trips. As more and more lines are tripped, power cannot be delivered to some loads. An imbalance between generation 
and loads [5,6] causes the system to enter a fast cascading period, when electrical instabilities such as transient instability and voltage instability are involved [7]. If corrective control actions are not taken or there is a failure to isolate the affected region to tackle these issues, an additional line trip may trigger oscillation in the system before its complete collapse [8]. Under such circumstances, and as a last resort, controlled islanding has been recommended to save the power system from a big blackout [2,9].

However, before any controlled islanding scheme [10-12] is executed, three areas of concern must be addressed:

Stage 1: When to island?

Stage 2: Where to island?

Stage 3: Dynamic stability evaluation in each island after islanding.

The first question is of crucial importance as islanding too early means an unnecessary, heavy disturbance, but islanding too late means the blackout will not be prevented from happening. Thus, early recognition and identification of "the point of no return" that leads to a blackout unless controlled islanding is executed, is of crucial importance. Despite its importance the issue has not attracted much research attention [13]. A trained decision tree (DT), based on off-line simulation data coming from one specific system was proposed in $[14,15]$ to decide when to island. Probability analysis based on measured data from previous blackout events was used in [16] to decide if the cascading failures will propagate in the network. This paper aims to answer the question, when is "the point of no return" by assessing if the next line that is due to be tripped due to overloads will cause the loss of dynamic stability. While a blackout is often caused by a combination of factors including transient instability and a voltage collapse, large power oscillations usually precede a blackout so they are a good indication of the "point of no return".

Obviously, blackouts can be caused not only by transient instability due to cascading line tripping. The procedure proposed in this paper is general and here, as the first step, we concentrate on checking the consequences of line trips on transient stability. Further research is needed to include an investigation of the consequences of generator trips and system collapse modes other than transient instability modes. This is the subject of our current research.

The "point of no return" must be assessed quickly as there is no time for time-consuming time-domain simulations to assess system stability. Hence, we have resorted to a fast, transient stability assessment using the single machine equivalent (SIME) method [17-19]. SIME was originally proposed to predict the effects of faults; however, in this paper we use it to predict if the loss of a line will cause transient instability. SIME firstly obtains two dynamic equivalent machines by transforming the multi-machine system, then a one machine infinite bus (OMIB) system can be obtained by further reduction [20]. Thus, the issue of transient stability is simplified to one single equation based on the well-known concept of equal area criterion. On-line application of SIME is dependent on real-time data based on WAMS and the SIME keeps monitoring the system status and calculates in advance whether transient instability is imminent by assessing the next line predicted to be tripped, which could lead to blackout.

If SIME predicts that the next line to be tripped will cause the system to lose stability, appropriate islands have to be identified that would prevent instability. This constitutes Stage 2 of the proposed methodology. There has been a significant research effort regarding the selection of appropriate islands for controlled islanding [21-23]. In [24-26], the ordered binary decision diagram (OBDD) method was proposed to satisfy different conditions, including easy synchronization and a good power balance in each island. In [27-29], a slow coherency-based method was used to provide islanding cutsets by identifying coherent generator groups first, and then searching for minimal cutsets with minimal PF imbalance in each controlled island [30]. An alternative is PF tracing [31] as it clusters the network into islands in such a way that power flowing in cutset lines is minimized. Hence, it minimizes power imbalances in each island while minimizing the shock to the system due to islanding itself. 
In this paper we use PF tracing [31] for identification of islands in Stage 2 but the proposed methodology is general and can be used with any other islanding scheme.

The next question to be answered is if the islands identified in Stage 2 will be stable. This constitutes Stage 3 of the proposed methodology. This is an important question as islanding itself constitutes a heavy disturbance to the system. We used SIME again for this purpose. If SIME indicated that one or more of the islands would be unstable, the islanding algorithm in Stage 2 was run again to identify different islands that would be transiently stable.

This paper provides a new and whole framework for a three-stage controlled islanding scheme to prevent blackouts. In addition, this paper concentrates on the first and third stages and uses SIME to assess transient stability which leads to blackouts. In our research, the novelty is that SIME has been modified and its application is extended to assess transient stability based on the power network topology change instead of the traditional fault applied on lines, which has not been studied in previous research. Power system blackouts occur due to a number of reasons, such as voltage collapse, frequency collapse and transient instability. In this paper we only investigate transient stability in the hypothesis of this research, which leads to blackouts during cascading outages. The study aims to find out if SIME can be used as a blackout predictor in terms of transient stability and how the aforementioned three steps are interconnected in order to execute successful islanding to prevent blackouts.

The proposed methodology is illustrated in the paper using a 10-machine, 39-bus dynamic New England power system model.

\section{Single Machine Equivalent Method}

The principle of the SIME method is to obtain an OMIB system from the reduction of two machine dynamic equivalents, which is received through the transformation of the multi-machine system [32]. Its assessment of transient stability is based on online data monitoring at continuous intervals.

Assessing SIME stability is dependent on the structure of the equivalent OMIB system as well as the $P-\delta$ curve of the OMIB system. When a serious fault happens in the power system, generators in the system form two groups and these two groups oscillate against each other. The group with increased generator angles are called the critical cluster, leaving the remaining generators, which are named non-critical. The structure of the OMIB system is determined by identifying the critical cluster of generators, which can be achieved by Taylor series expansion. In addition, weighted least-squares (WLS) estimation is used to predict the OMIB system's $P-\delta$ curve by successively updating dynamic generator angles during the dynamic change after line contingencies, thus, it ensures the assessment accuracy of transient stability. Based on this critical cluster concept, Taylor series and Quant can be used to predict and plot the generator angle trajectories when system operating conditions change, based on the initial generator angle data obtained from online WAMS.

\subsection{Model of Multi-Machine Power System}

For a power system with $n$ generators, the electrical power and motion are presented in [33-35] as follows for the $i$ th generator:

$$
\begin{gathered}
M_{i} \ddot{\delta}_{i}=P_{m i}-P_{e i} i=1,2, \ldots, n \\
P_{e i}=E_{i}^{2} Y_{i i} \cos \theta_{i i}+\sum_{j=1, j \neq i}^{n} E_{i} E_{j} Y_{i j} \cos \left(\delta_{i}-\delta_{j}-\theta_{i j}\right)
\end{gathered}
$$

where $M_{i}, P_{m i}$ and $E_{i}$ are supposed to be constant values. In addition, the loads are considered as the constant impedances. 


\subsection{Model of Equivalent Two-Machine Power System}

Two assumptions are made in the SIME method in order to transform a multi-machine system to an equivalent two-machine system. One is that generator angles under severe contingency situations move apart to form two groups. Transient stability in multi-machine systems is controlled by those generators that are responsible for the irrevocable system separation whenever the equivalent generator angle of those machines passes its corresponding unstable equilibrium point. Those generators are called critical generators or a critical cluster, although sometimes the critical generator is a just single generator. The other assumption is that each group's generator angles can be modeled using the center of angles (COA). Based on that assumption, the corresponding two-machine equivalent system is modeled as follows.

The motion for cluster $A$ along with electrical power $P_{e l}$ for the $l$ th generator of cluster $A$ are represented by:

$$
\begin{gathered}
M_{a} \ddot{\delta}_{a}=\sum_{l \in A}\left(P_{m l}-P_{e l}\right) \\
P_{e l}=E_{l}^{2} Y_{l l} \cos \theta_{l l}+\sum_{j=1, j \neq i}^{n} E_{l} E_{a} Y_{l a} \cos \left(\delta_{a}-\delta_{s}-\theta_{l s}\right)+\ldots+\sum_{j \in A, j \neq l} E_{l} E_{j} Y_{l j} \cos \theta_{l j} ; \forall l \in A, \forall j \in A
\end{gathered}
$$

The motion for cluster $S$ along with the electrical power of generators in cluster $S$ are in the same form as Equations (3) and (4) if critical generators are a group of machines; the electrical power $P_{e s}$ of $S$ can be represented as Equation (5) if the critical generator is a machine only.

$$
P_{e s}=E_{s}^{2} Y_{s S} \cos \theta_{s s}+\sum_{j \in A} E_{s} E_{j} Y_{s j} \cos \left(\delta_{s}-\delta_{a}-\theta_{s j}\right)
$$

\subsection{Model of Equivalent One Machine Infinite Bus Power System}

The motion of the OMIB equivalent system can be given using relative rotor angle along with rotor acceleration between two generator groups $S$ and $A$.

$$
M \ddot{\delta}=P_{m}-\left[P_{C}+P_{M A X} \sin (\delta-v)\right]
$$

where $P_{C}$ and $P_{M A X}$, denote the equivalent mechanical input and electrical power; $v, C$ and $D$ are the derived coefficients represented via mathematical transformation from the two-machine equivalent to an OMIB equivalent, which are shown as follows:

$$
\begin{gathered}
P_{C}=\left(M_{a} E_{s}^{2} G_{s s}-M_{s} \sum_{l, j \in A} E_{l} E_{j} G_{l j}\right) M_{T}^{-1} \\
P_{M A X}=\left(C^{2}+D^{2}\right)^{1 / 2}, v=\tan ^{-1}(C / D) \\
C=\left(M_{a}-M_{s}\right) M_{T}^{-1} \sum_{l \in A} E_{s} E_{l} G_{s l} \\
D=\sum_{l \in A} E_{s} E_{l} B_{s l}
\end{gathered}
$$

\subsection{Critical Machine Ranking}

Critical machine ranking (CMR) in $[36,37]$ is used in order to help identify critical generators under contingency. With the help of CMR, the multi-machine system can be transformed into the OMIB equivalent system.

Truncated Taylor series expansion as shown in Equation (11) is adopted in every generator's angle revolution if any concerning outages occur in the system. A large constant step size of time 
(i.e., $0.1 \mathrm{~s}$ ) is chosen between the starting point and the time when the oscillation fades away after breaker operation at $t\left(\delta_{0}^{+}\right)$.

$$
\delta_{t}=\delta_{o}+\gamma \frac{t^{2}}{2}+\gamma^{(2)} \frac{t^{4}}{24}+\gamma^{(4)} \frac{t^{6}}{720}+\ldots
$$

where $\gamma$ represents an acceleration at the time $t=t\left(\delta_{0}^{+}\right)$:

$$
\gamma_{i}=\left[P_{m i}-P_{e i}\left(\delta\left(t_{o}^{+}\right)\right)\right] M_{i}^{-1}
$$

In Equation (11), the successive derivatives $\gamma^{(2)}$ and $\gamma^{(4)}$ are obtained at the time $t\left(\delta_{0}^{+}\right)$and $\delta_{0}$ denotes the original steady state rotor angle. The trajectories of each individual generator angle can be plotted using this Taylor-series-expansion-based CMR. Critical machines can be observed and identified when the predictive generator angle trajectory reaches the unstable equilibrium point. Then, the machines are sorted in decreasing order of their generator angles. The generators above the gap between two consecutive maximum angles of the generators are determined as critical generators. This signal can be obtained very quickly via the computing of Equation (11) in order for SIME to calculate the stability margin.

\subsection{Steps of the Single Machine Equivalent Method}

1) Once the line outage happens at $t_{e}$, online measurements start collecting data at a time $t_{i}$ shortly after the outage, each generator angles at times $t_{i}-2 \Delta t, t_{i}-\Delta t$ and $t_{i}$ are obtained. Then, based on the Taylor-series-extension method, the future generator angle trajectories of each generator are predicted and identify the candidate critical generators.

2) The OMIB equivalent system is constructed once the critical generators are identified. With three sets of data regarding generator angle and electrical power for each generator, three sets of data for the OMIB equivalent system can also be obtained at times $t_{i}-2 \Delta t, t_{i}-\Delta t$ and $t_{i}$. Therefore, the three coefficients of $a, b, c$ in Equation (13) are calculated using quadratic function 20 to approximate the $P_{a}-\delta$ curve in Figure 1:

$$
\hat{P}_{a}(\delta)=a \delta^{2}+b \delta+c
$$

With these coefficients gained according to three data sets from the online measurement, the $P_{a}-\delta$ curve is obtained.

3) The OMIB unstable equilibrium angle $\delta_{u}$ is calculated with the coefficients in Equation (13) found in the last step.

4) Then, the stability margin $\eta$ can be computed by Equation (14), which indicates the difference between potential energy and kinetic energy.

$$
\eta=-\int_{\delta_{i}}^{\delta_{u}} P_{a} d \delta-\frac{1}{2} M \omega_{i}^{2}
$$

5) If $\eta$ is close to zero or negative, then an unstable system can be determined and further actions need to be taken.

6) If the unstable system is determined, the time left before instability $t_{u}$ can be obtained using Equation (15).

$$
t_{u}=t_{i}+\int_{\delta_{i}}^{\delta_{u}}\left(\frac{d \delta}{\sqrt{\frac{2}{M} \int_{\delta_{i}}^{\delta}-P_{a} d \delta+\omega_{i}^{2}}}\right)
$$

7) Continue updating system status in terms of transient stability through assessing three new sets of the data from online measurement at consecutive time intervals $\Delta t$. 


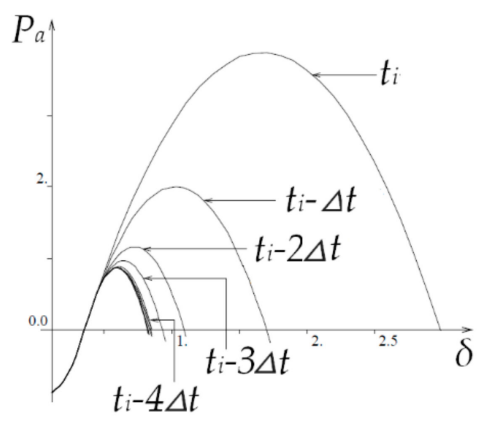

Figure 1. Refreshing transformed one machine infinite bus (OMIB) system's $P_{a^{-}} \delta$ curves.

\section{Application of Single Machine Equivalent in the Preventive Islanding Scheme}

\subsection{Three-Stage Procedure for Preventing Wide-Area Blackouts}

A successful controlled islanding scheme not only has to consider the dynamic state before islanding, but also requires a secure state in each islanded system after splitting. The flowchart of the proposed three-stage scheme is shown in Figure 2.

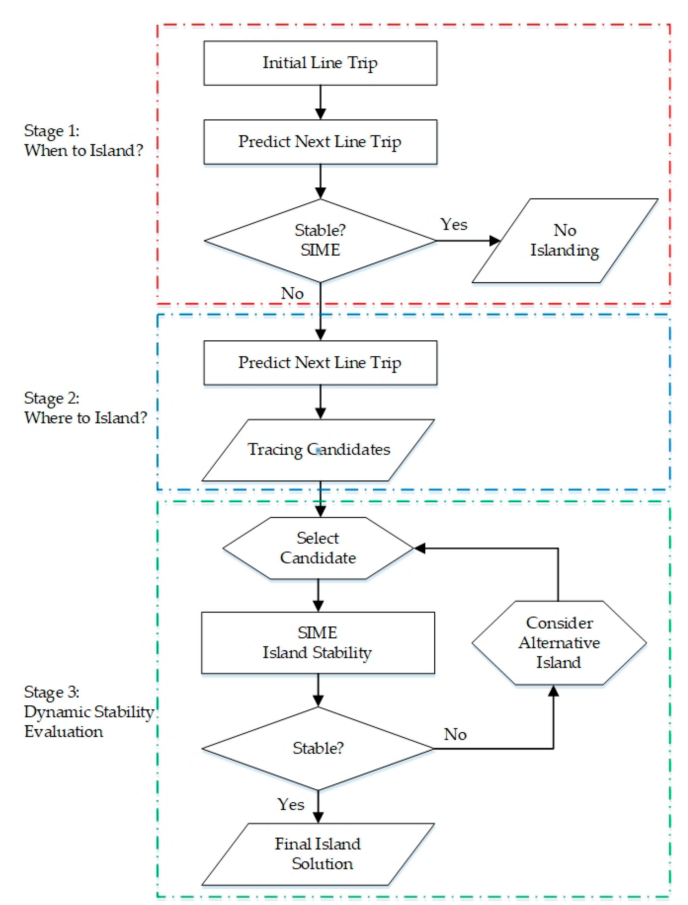

Figure 2. Flowchart of three-stage strategies for controlled islanding.

This paper concentrates on Stages 1 and 3. Stage 2, i.e., islanding methodology itself, is independent of Stages 1 and 3 and in this paper we have used, just as an example, PF tracing to identify suitable islands. Alternatively, any other islanding method proposed in the literature can be used.

\subsection{Stage 1: When to Island?}

Using SIME, transient stability can be assessed during cascading outages and a decision can be taken about "when to island". The flowchart of this stage is shown in Figure 3.

Once a first line is tripped due to a fault, online transient stability assessment is immediately triggered for prediction of the transient stability state using the assumption that the reconfiguration of flows causes another line to be tripped. In this period, PF runs based on online data from WAMS. Based on the thermal thresholds of the lines or the setting of distance protection, the next expected 
tripped line can be identified. Then, time domain simulation (TDS) starts running for about $0.1 \mathrm{~s}$ assuming that the next expected line does trip, in order to obtain the initial generator angles and the required calculation data, such as electrical power and generator angular velocity shown by Equation (14), which are used to calculate the stability margin. Also based on these initial generator angles, CMR can identify the critical cluster of the machine(s) using Equation (11). If the computed stability margin is positive or indicates stable operation, the system is assumed to remain stable so that no controlled islanding is necessary. The diagnostic system will then wait until the next contingency happens. If the computed stability margin is negative or indicates unstable operation, Stage 2 is activated. SIME is used to calculate the time left to instability and the scheme proceeds to Stage 2.

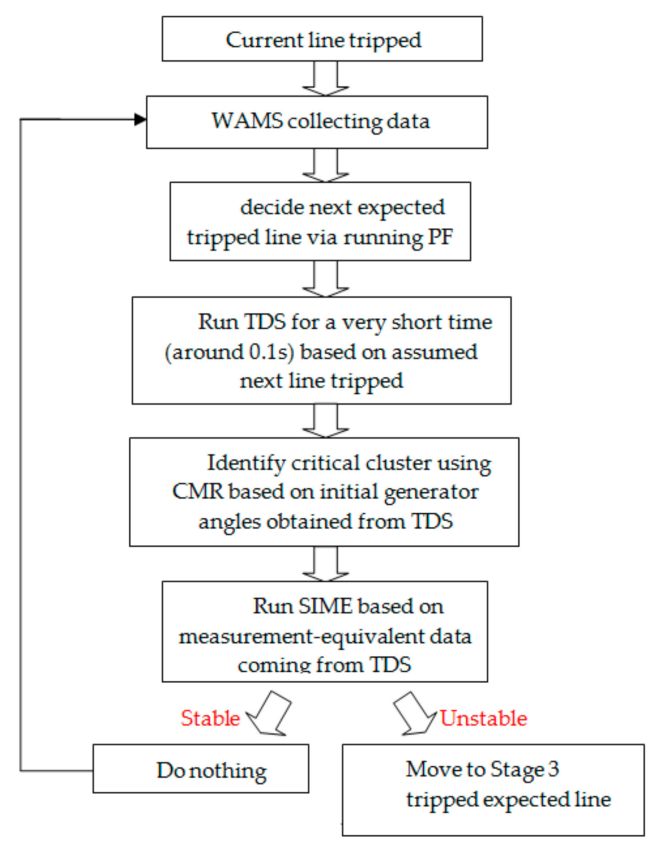

Figure 3. Flowchart of applying single machine equivalent (SIME) online to determine when to island.

The main reason for using SIME rather than TDS for transient stability assessment is its speed. As transient instability can happen within a few seconds after a severe disturbance, saving time is a priority in order to leave enough time for the system operator to respond and take control actions such as controlled islanding. Secondly, the stability margin provided by SIME can help the system operator be alert regarding transient stability moving towards the instability boundary and to be aware of how far away the system is from transient instability after the next line trip.

Obviously, blackouts can be caused not only by transient instability triggered by cascading line tripping. The procedure proposed in this paper is general and here, as the first step, we concentrate on checking the consequences of line trips on transient stability. Further research is needed to include the consequences of generator trips and also system collapse modes other than transient instability. This is the subject of our current research.

\subsection{Stage 2: Where to Island?}

In Stage 2, an islanding algorithm is used to identify islands that, when disconnected from each other, prevent a blackout. In this paper, we use PF tracing [31] as an example but any other islanding scheme could be used.

Tracing attempts to isolate the area where a disturbance started. To do this, it identifies closely connected nodes by calculating the contributions of power flowing through a given node to all the other nodes upstream and downstream in the directed graph of flows. If the contributions are below a chosen threshold value, the nodes are deemed to be closely connected and should remain in one island. Hence, choosing a different threshold value will result in different islands, and if a particular 
islanding scheme is found to be transiently unstable, an alternative islanding can be chosen based on a different threshold value.

Tracing-based islanding has the advantage that it attempts to find such cutset lines connecting the islands that power flowing through them is minimized. This has the effect of maximizing the power balance in each island and minimizing the shock to the system caused by islanding itself, therefore, helping to maintain dynamic stability.

\subsection{Stage 3: Dynamic Stability Evaluation}

In Stage 3, we investigated whether a selected islanding candidate was transiently stable after islanding. This step is necessary for those islanding methodologies applied in Stage 2 that do not directly include transient stability analysis. On the other hand, for example, if the slow-coherency methodology is used to select islands, Stage 3 may be omitted as avoiding dynamic instability is the main purpose of applying the slow-coherency method.

In this stage, we also applied SIME for fast transient stability assessment. If the analysis concludes that one of the islands would be unstable following controlled islanding, different islands have to be chosen. In the case of the tracing methodology that we applied, finding alternative islands is possible by changing the threshold value of the contributions or changing the weights associated with different decision factors. Further details can be found in [38]. SIME will then continue to assess the stability of the newly-provided islands until they prove to be transiently stable. If no stable islands can be found, additional actions have to be undertaken such as generator tripping in generation-rich islands and load shedding in islands with generation deficit. Here SIME can also be used to identify which generation or load to shed. Details can be found in $[17,18,20]$.

It should be noted that time is of the essence in any controlled islanding scheme, therefore, the identification of candidate islands should be ideally be done a-priori. Use of SIME makes it possible for the whole three-stage process to be executed quickly before a blackout happens.

\section{Case Study}

An IEEE 39-bus power system with 10 generators [39] as shown in Figure 4, was adopted to demonstrate the performance of the methodology. It includes transient stability prediction using SIME to identify "the point of no return" for islanding decision making, and SIME is also used to assess the transient stability of the selected islands. The IEEE 39-bus New England system with classical models of generators was built in the power system assessment tool (PSAT). For the studied case, the time-domain simulation results are also shown in order to check the SIME calculation results.

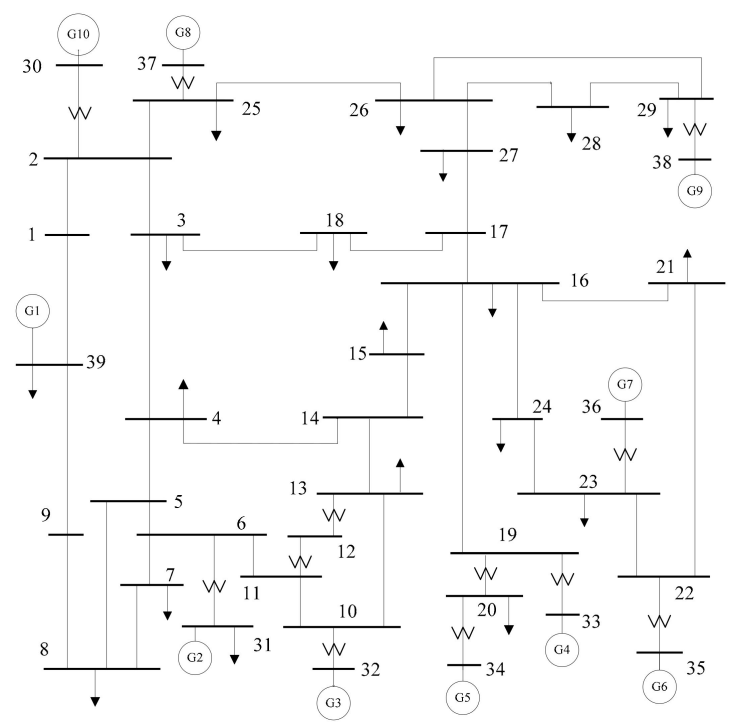

Figure 4. Ten-generator 39-bus New England system. 
In order to stress the system model to simulate a cascading environment, PQ loads were increased up to 1.14 times the original level. To simulate a cascade, two neighboring lines 4-5 and 4-14 were tripped in $2 \mathrm{~s}$ intervals until transient instability occurred. Online SIME application for transient stability assessment not only relies on WAMS measurement for PF calculation, but also needs post-line-trip data for transient stability prediction. These input data, such as generator angle and generator electrical power, come from running TDS for $0.1 \mathrm{~s}$, assuming that the next expected line actually tripped. The other input data that SIME needs to help transform the multi-machine system to an OMIB system is a reduced system admittance matrix. This changes with the change of network topology once the line is tripped off. This reduced system admittance matrix can be obtained through calculation using the network data and it is independent of both the WAMS measurement and time-domain simulation.

\subsection{Transiently Stable Case}

After Line 4-5 was tripped, TDS runs for $0.1 \mathrm{~s}$, which is approximately equivalent to $1 \mathrm{~s}$ in real-time. The diagnostic system started collecting data $100 \mathrm{~ms}$ after the contingency in order to wait for the initial oscillation to die out. The rate of collecting data was 20 ms and SIME started assessing transient stability status in $140 \mathrm{~ms}$ after the line contingency. The unstable equilibrium angle was initially estimated by SIME to be $1.945 \mathrm{rad}$ giving a positive stability margin of $2.64(\mathrm{rad} / \mathrm{s})^{2}$. Those values were continuously updated resulting in $1.768 \mathrm{rad}$ and $2.05(\mathrm{rad} / \mathrm{s})^{2}$, respectively, after $740 \mathrm{~ms}$. This means that the potential energy was bigger than kinetic energy and the system was still transiently stable.

During a stable scenario, the critical generator(s) do not exist as generator angles are oscillating instead of splitting apart from each other. However, based on oscillation groups, which are G2, G3 in one group and the remaining ones in another group, the system can still be transferred to a corresponding OMIB system to calculate the stability margin. The calculation results were confirmed by time-domain simulations, which are represented in Figure 5.

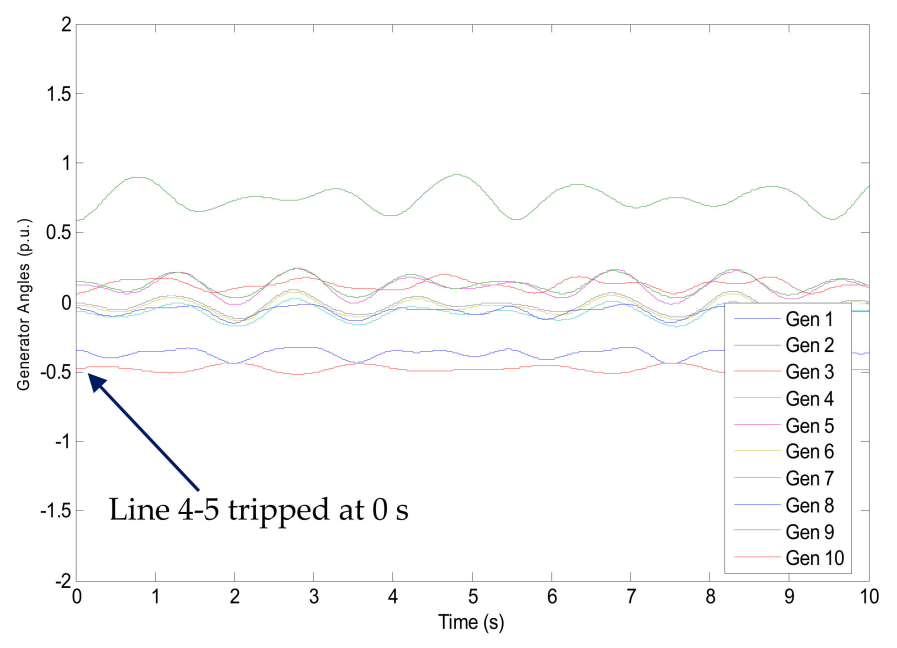

Figure 5. Transiently stable case after Line $4-5$ was tripped.

\subsection{Transiently Unstable Case}

Next, we assumed that a neighboring Line 4-14 was tripped by protection $2 \mathrm{~s}$ after Line 4-5. Calculations were undertaken every $20 \mathrm{~ms}$ though the results shown in Table 1 are for every 4 th time step. The meaning of the columns is described in their headings.

Generator G2 was identified as a critical generator. Table 1 shows that the stability margins are initially close to zero and gradually become negative from $560 \mathrm{~ms}$. Once the stability margin is negative or close to zero, the system can be declared transiently unstable. The calculation results were confirmed by time-domain simulations as shown in Figure 6. After the second Line 4-14 was tripped 
at $2 \mathrm{~s}$, the generator angles did not split immediately and significantly into two groups. This can only be observed from around $1.5 \mathrm{~s}$ until $3.5 \mathrm{~s}$. During this period, multi swings might occur in the system until the oscillation leads to asynchronism. This could explain why initially there were no negative stability margins obtained during the swing period.

Note that the third column of Table 1 contains an estimate of critical islanding time (CIT), i.e., time left for executing preventive islanding if the system is to remain stable. The longer the CIT, the better.

Table 1. Transiently stable indications after line $4-5$ and $4-14$ were tripped.

\begin{tabular}{cccc}
\hline $\begin{array}{c}\text { Time after Contingency } \\
\text { (ms) }\end{array}$ & $\begin{array}{c}\text { Unstable Equilibrium } \\
\text { Angle (rad) }\end{array}$ & $\begin{array}{c}\text { Time Left to Instability } \\
\text { after Tripping Line 4-14 (ms) }\end{array}$ & $\begin{array}{c}\text { Stability Margin } \\
\text { (rad/s) })^{\mathbf{2}}\end{array}$ \\
\hline 140 & 1.581 & 406 & 0.19 \\
220 & 1.580 & 475 & 0.17 \\
300 & 1.579 & 540 & 0.14 \\
380 & 1.579 & 601 & 0.10 \\
460 & 1.579 & 657 & 0.06 \\
540 & 1.579 & 707 & 0.01 \\
620 & 1.580 & 747 & -0.05 \\
700 & 1.580 & 773 & -0.09 \\
\hline
\end{tabular}

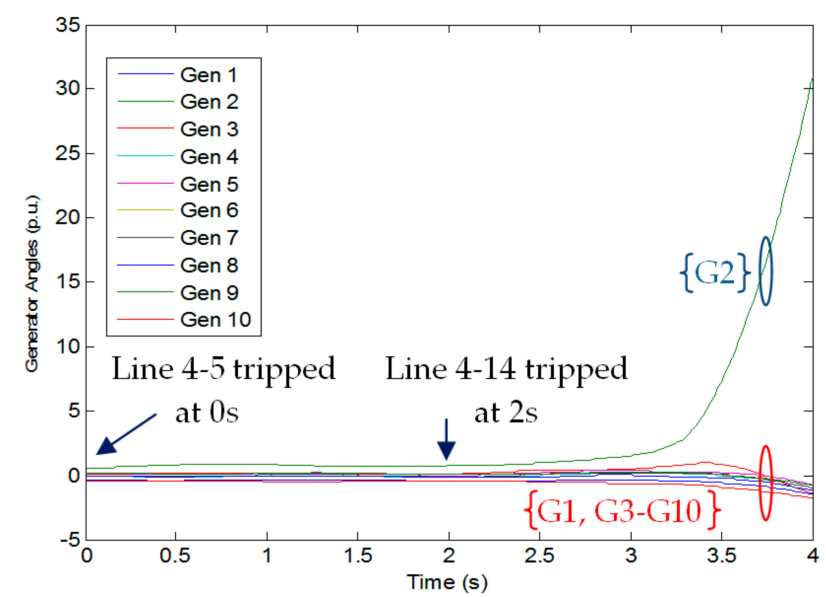

Figure 6. Transiently unstable case after Line 4-5 and 4-14 were tripped.

\subsection{Assessment of Transient Stability of the Islands}

Now we will illustrate how SIME can be used with tracing-based islanding methodology [31] to assess the transient stability of the selected islands.

Once the second Line 4-14 was assumed to be tripped and SIME indicated the system was going to lose synchronism, the PF tracing-based method [31] was used to identify possible islands in Stage 2 of the methodology. The details are beyond the scope of this paper. Dashed lines in Figure 4 show the borders of the resulting island denoted as IS1 and indicate that the cutset consists of Lines 9-39, 3-4 and 15-16.

Once an islanding scheme has been identified, SIME can be used to predict if the system will be transiently stable following islanding (Stage 3 of the methodology) assuming that islanding is executed before the critical islanding time (CIT shown in the third column in Table 1). In other words, islanding was assumed to be executed $0.77 \mathrm{~s}$ after tripping Line 4-14 in order to assess the transient stability status in each island. The results indicate that the islands are indeed stable but these are not shown in full due to lack of space. The stability margin for the first IS1 island was found initially (at $140 \mathrm{~ms}$ after islanding) to be $7.01(\mathrm{rad} / \mathrm{s})^{2}$ reducing to $2.59(\mathrm{rad} / \mathrm{s})^{2}$ after $740 \mathrm{~ms}$. The unstable equilibrium angle was initially 3.099 rad reducing to $2.995 \mathrm{rad}$ after $740 \mathrm{~ms}$. The second, larger island was found to be very stable with the stability margin increasing from the initial14 (rad/s) ${ }^{2}$ to 160 (rad/s) $)^{2}$ after $740 \mathrm{~ms}$.

Figure 7 shows the results of the time-domain simulation, which confirmed the SIME predictions when islanding was executed $0.9 \mathrm{~s}$ after Line 4-14 tripped (2.9 s after the start of simulations in 
Figure 7). After splitting the network at $2.9 \mathrm{~s}$, generator angles separated into two groups to form two islands. One island contained two generators $\{\mathrm{G} 2, \mathrm{G} 3\}$ while the other island contained the remaining generators $\{\mathrm{G} 1, \mathrm{G} 4, \mathrm{G} 5, \mathrm{G} 6, \mathrm{G} 7, \mathrm{G} 8, \mathrm{G} 9, \mathrm{G} 10\}$. The two islands remained stable because the generator angles stayed close to each other in both islands, although, obviously both groups of angles separated following islanding.

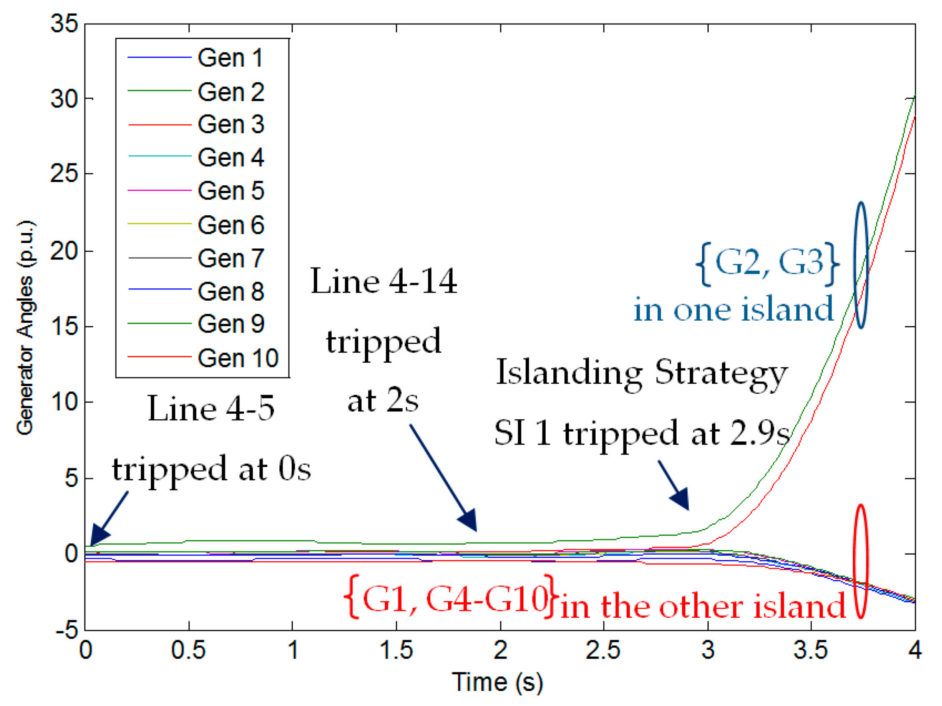

Figure 7. Islanding strategy IS1 applied at critical islanding time (CIT) $2.9 \mathrm{~s}$.

Note that the islanding time of $0.9 \mathrm{~s}$ is quite close to the maximum (critical) islanding time of $0.77 \mathrm{~s}$ identified in Table 1 using SIME.

Figure 8 illustrates the results of the time-domain simulations when the islanding strategy IS1 split the network into two islands at 2.95 s, i.e., $0.95 \mathrm{~s}$ after Line $4-14$ tripped. As a result, G2 and G3 lost synchronism as the islanding was undertaken too late. That loss of stability was predicted using SIME, as shown in Table 2. Table 2 shows that calculated stability margins are initially close to zero and then become negative from the fourth updating.

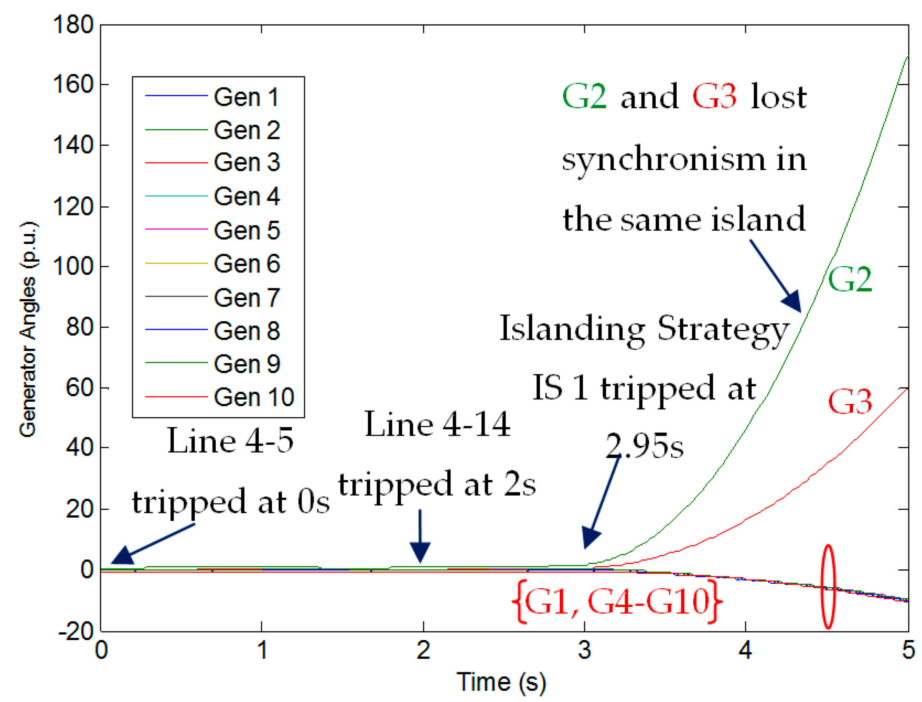

Figure 8. Islanding strategy IS1 applied beyond critical islanding time at $2.95 \mathrm{~s}$. 
Table 2. Transiently stable indications in the island containing G2 and G3 after islanding strategy IS1 was undertaken.

\begin{tabular}{cccc}
\hline $\begin{array}{c}\text { Time after Contingency } \\
(\mathbf{m s})\end{array}$ & $\begin{array}{c}\text { Unstable Equilibrium } \\
\text { Angle (rad) }\end{array}$ & $\begin{array}{c}\text { Time Left to Instability } \\
\text { after Islanding IS1 (ms) }\end{array}$ & $\begin{array}{c}\text { Stability Margin } \\
\text { (rad/s) } \mathbf{~}^{2}\end{array}$ \\
\hline 140 & 2.959 & 1121 & 2.67 \\
200 & 2.996 & 1172 & -1.02 \\
280 & 3.101 & 1226 & -3.68 \\
360 & 6.286 & -12.9 \\
440 & 6.316 & 1391 & -3.74 \\
520 & 9.253 & 1494 & -0.24 \\
620 & 12.49 & -1609 & -9.12 \\
660 & 12.60 & 1693 & -3.74 \\
740 & 15.65 & & -3.70 \\
\hline
\end{tabular}

\subsection{Executation Time for the Three-Stage Procedure}

All the analysis and calculation involved in the proposed 3-stage procedure should take as little time as possible in order to leave enough time for the islanding itself. This is the reason we used SIME as it only has to calculate the stability margin based on the transformed OMIB system. The time consumed by using SIME in both of Stage 1 and Stage 3 to predict transient stability was $0.33 \mathrm{~s}$ using Intel Core $2.93 \mathrm{GHz}$ processor with $4 \mathrm{~GB}$ of RAM. This includes running PF ( $0.03 \mathrm{~s})$ to identify the next expected tripped line, short-time TDS $(0.1 \mathrm{~s})$ based on the assumption that the next line is tripped and running SIME codes $(0.2 \mathrm{~s})$ to obtain the stability margin. In addition, the time used for obtaining islanding cutsets was $0.04 \mathrm{~s}$, which includes finding islanding candidates based on tracing and identifying best islanding solution through optimization in terms of minimal PF disruption. Thus, the total time consumed in this three-stage controlled islanding was $0.37 \mathrm{~s}$, which is less than the $0.9 \mathrm{~s}$ identified as the time margin, i.e., CIT.

\section{Discussion}

In terms of "when to island" in this controlled islanding scheme, SIME was applied to assess the transient stability before imminent blackout to determine the appropriate time to split the network. Compared with the previous DT mathematical method [14,15], SIME is an engineering method for this controlled islanding scheme, which can be generally applied in every power network. The DT method cannot give the solution for when to island, and cannot guarantee the stability of the split islands. However, the proposed method can provide the answer for when to island, and guarantees the stability of the split islands. Thus, it can be concluded that the proposed method is better than the previous DT mathematical method. The challenge of the proposed method is that there is a compromise between the accuracy and calculation speed, and this needs to be further investigated in future research. In addition, a larger power system network needs to be adopted in the case study using SIME in this controlled islanding scheme.

\section{Conclusions}

This paper has proposed a framework for an adaptive three-stage controlled islanding scheme as a last resort of action to prevent wide-area blackouts. In addition, SIME and PF tracing methods were adopted in this research. In Stage 1, SIME was used to determine when to island in order to prevent transient instability and it was also used to evaluate the dynamic stability in each island identified by an islanding scheme in Stage 3. In Stage 2, the PF tracing method was used to help find the expected islanding cutsets. In the last section, an IEEE 10-generator 39-bus system was used to test this three-stage controlled islanding scheme during cascading line outages. The calculation of transient stability using SIME was very fast and also reasonably accurate, as confirmed by time-domain simulation results. 
Author Contributions: H.S. and L.Y. conceptualized the study; H.S. and C.H. performed the analyses; Y.M., Y.L. and W.L. acquired funding; H.S. and B.Z. performed investigations; S.S., P.J. and F.M. acquired resources; H.S. and B.Z. wrote the original draft; and L.Y., C.H. and B.Z. reviewed and edited the manuscript.

Funding: This work was supported by the State Grid Henan Electric Power Company Grant Number 5217L0170010.

Acknowledgments: The authors would like to thank Prof. Janusz Bialek and Dr. Seán Norris for their assistance and the Engineering and Physical Science Research Council (EPSRC) Grant EP/G060169/1 for support.

Conflicts of Interest: The authors declare no conflict of interest.

\section{Nomenclature}

$\begin{array}{ll}\delta_{\mathrm{i}} & \text { Rotor angle } \\ \mathrm{M}_{\mathrm{i}} & \text { Inertia coefficient } \\ \mathrm{P}_{\mathrm{mi}}\left(\mathrm{P}_{\mathrm{ei}}\right) & \text { Mechanical input (electrical power) } \\ \mathrm{E}_{\mathrm{i}} & \text { Potential behind transient reactance of } d \text {-axe } \\ \mathrm{Y} & \text { Reduced matrix of admittance to the node of generators } \\ \theta_{\mathrm{ij}}\left(\mathrm{Y}_{\mathrm{ij}}\right) & \text { Argument (Modulus) of the } i \text { th row and the } j \text { th column element of } Y \\ \mathrm{~S} & \text { The critical machines' set } \\ \mathrm{S} & \text { The aggregated machine of } S \\ \mathrm{~A} & \text { The set of non-critical machines } \\ \mathrm{a} & \text { The aggregated machine of } A \\ \mathrm{~B} & \text { Susceptance } \\ \mathrm{G} & \text { Conductance }\end{array}$

\section{References}

1. Han, C.; Zhao, Y.; Lin, Z.; Ding, Y.; Yang, L.; Lin, G.; Mo, T.; Ye, X. Critical lines identification for skeleton-network of power systems under extreme weather conditions based on the modified VIKOR method. Energies 2018, 11, 1355. [CrossRef]

2. Yang, B.; Vittal, V.; Heydt, G.T. Slow-coherency-based controlled islanding-A demonstration of the approach on the August 14, 2003 blackout scenario. IEEE Trans. Power Syst. 2006, 21, 1840-1847. [CrossRef]

3. Liscouski, B.; Elliot, W. Final Report on the August 14, 2003 Blackout in the United States and Canada; US-Canada Power System Outage Task Force; Office of Electricity: Washington, DC, USA, 2004.

4. Xue, Y.S. Towards space-time cooperative defence framework against blackouts in China. In Proceedings of the 2007 IEEE Power Engineering Society General Meeting, Tampa, FL, USA, 24-28 June 2007.

5. Hui, H.X.; Ding, Y.; Liu, W.D.; Lin, Y.; Song, Y.H. Operating reserve evaluation of aggregated air conditioners. Appl. Energy 2017, 196, 218-228. [CrossRef]

6. Xie, D.J.; Hui, H.X.; Ding, Y.; Lin, Z.Z. Operating reserve capacity evaluation of aggregated heterogeneous TCLs with price signals. Appl. Energy 2018, 216, 338-347. [CrossRef]

7. Henneaux, P.; Labeau, P.; Maun, J. Blackout PRA based identification of critical initial conditions and contingencies. In Proceedings of the IEEE International Conference on Probabilistic Methods Applied to Power Systems, Istanbul, Turkey, 10-14 June 2012.

8. Lin, Z.Z.; Wen, F.S.; Wang, H.F.; Lin, G.Q.; Mo, T.W.; Ye, X.J. CRITIC-based node importance evaluation in skeleton network reconfiguration of power grids. IEEE Trans. Circuits Syst. II Express Briefs 2018, 65, $206-210$. [CrossRef]

9. Goo, B.; Hur, J. Estimation for expected energy not served of power systems using the screening methodology of cascading outages in South Korea. Energies 2018, 11, 81. [CrossRef]

10. Arraño-Vargas, F.; Rahmann, C.; Valencia, F.; Vargas, L. Active splitting in longitudinal power systems based on a WAMPC. Energies 2018, 11, 51. [CrossRef]

11. Lin, Z.Z.; Wen, F.S.; Zhao, J.H.; Xue, Y.S. Controlled islanding schemes for interconnected power systems based on coherent generator group identification and wide-area measurements. J. Mod. Power Syst. Clean Energy 2016, 4, 440-453. [CrossRef]

12. Song, H.L.; Wu, J.Y.; Wu, K. A wide-area measurement systems-based adaptive strategy for controlled islanding in bulk power systems. Energies 2014, 7, 2631-2657. [CrossRef] 
13. Lin, Z.Z.; Wen, F.S.; Xue, Y.S. A restorative self-healing algorithm for transmission systems based on complex network theory. IEEE Trans. Smart Grid 2016, 7, 2154-2162. [CrossRef]

14. Senroy, N.; Heydt, G.T.; Vittal, V. Closure to discussion of "decision tree assisted controlled islanding". IEEE Trans Power Syst. 2006, 4, 1790-1797. [CrossRef]

15. Mei, K.J.; Rovnyak, S.M. Response-based decision trees to trigger one-shot stabilizing control. IEEE Trans. Power Syst. 2004, 19, 531-537. [CrossRef]

16. Dobson, L.; Wierzbicki, K.R.; Carreras, B.A.; Lynch, V.E.; Newman, D.E. An estimator of propagation of cascading failure. In Proceedings of the Thirty-ninth Hawaii International Conference on System Science, Kauai, HI, USA, 4-7 January 2006.

17. Glavic, M.; Ernst, D.; Ruiz-Vega, D.; Wehenkel, L.; Pavella, M. E-SIME-A method for transient stability closed-loop emergency control: Achievements and prospects. In Proceedings of the 2007 iREP Symposium-Bulk Power System Dynamics and Control-VII, Charleston, SC, USA, 19-24 August 2007.

18. Bettiol, A.L.; Wehenkel, L.; Pavella, M. Transient stability-constrained maximum allowable transfer. IEEE Trans. Power Syst. 1999, 14, 654-659. [CrossRef]

19. Bettiol, A.L.; Zhang, Y.; Wehenkel, L.; Pavella, M. Transient stability investigations on a Brazilian network by SIME. In Proceedings of the 4th International Conference on Advances in Power System Control, Operation and Management, APSCOM-97, Hong Kong, China, 8-11 November 1997.

20. Pavella, M.; Ernst, D.; Ruiz-Vega, D. Transient Stability of Power Systems: A Unified Approach to Assessment and Control; Kluwer Academic Publishers: Dordrecht, The Netherlands, 2000.

21. Lin, Z.Z.; Wen, F.S.; Ding, Y.; Xue, Y.S. Wide-area coherency identification of generators in interconnected power systems with renewables. IET Gener. Transm. Distrib. 2017, 11, 4444-4455. [CrossRef]

22. You, H.B.; Vittal, V.; Wang, X.M. Slow coherency-based islanding. IEEE Trans. Power Syst. 2004, 19, 483-491. [CrossRef]

23. Ding, L.; Gonzalez-Longatt, F.M.; Wall, P.; Terzija, V. Two-step spectral clustering controlled islanding algorithm. IEEE Trans. Power Syst. 2013, 28, 75-84. [CrossRef]

24. Sun, K.; Zheng, D.Z.; Lu, Q. Splitting strategies for islanding operation of large-scale power systems using OBDD-based methods. IEEE Trans. Power Syst. 2003, 18, 912-923. [CrossRef]

25. Zhao, Q.C.; Sun, K.; Zheng, D.Z.; Ma, J.; Lu, Q. A study of system splitting strategies for island operation of power system: A two-phase method based on OBDDs. IEEE Trans. Power Syst. 2003, 18, 1556-1565. [CrossRef]

26. Sun, K.; Zheng, D.Z.; Lu, Q. A simulation study of OBDD-based proper splitting strategies for power systems under consideration of transient stability. IEEE Trans. Power Syst. 2005, 20, 389-399. [CrossRef]

27. Lin, Z.Z.; Wen, F.S.; Ding, Y.; Xue, Y.S.; Liu, S.Y.; Zhao, Y.X.; Yi, S.M. WAMS-based coherency detection for situational awareness in power systems with renewables. IEEE Trans. Power Syst. 2018, 33, 5410-5426. [CrossRef]

28. Xu, G.Y.; Vittal, V. Slow coherency based cutset determination algorithm for large power systems. IEEE Trans. Power Syst. 2010, 25, 877-884. [CrossRef]

29. Jin, M.; Sidhu, T.S.; Sun, K. A new system splitting scheme based on the unified stability control framework. IEEE Trans. Power Syst. 2007, 22, 433-441. [CrossRef]

30. Lin, Z.Z.; Wen, F.S.; Ding, Y.; Xue, Y.S. Data-driven coherency identification for generators based on spectral clustering. IEEE Trans. Ind. Inform. 2018, 14, 1275-1285. [CrossRef]

31. Norris, S.; Guo, S.; Bialek, J. Tracing of power flows applied to islanding. In Proceedings of the General Meeting of the IEEE-Power-and-Energy-Society, San Diego, CA, USA, 22-26 July 2012.

32. Ernst, D.; Ruiz-Vega, D.; Pavella, M.; Hirsch, P.; Sobajic, D. A unified approach to transient stability contingency filtering, ranking and assessment. IEEE Trans. Power Syst. 2001, 16, 435-443. [CrossRef]

33. Xue, Y.; Cutsem, T.V.; Ribbens-Pavella, M. A simple direct method for fast transient stability assessment of large power systems. IEEE Trans. Power Syst. 1988, 3, 400-412. [CrossRef]

34. Xue, Y.; Cutsem, T.V.; Ribbens-Pavella, M. Extended equal area criterion, justification, generalizations and application. IEEE Trans. Power Syst. 1989, 4, 44-52. [CrossRef]

35. Shao, H.B.; Norris, S.; Lin, Z.Z.; Bialek, J. Determination of when to island by analysing dynamic characteristics in cascading outages. In Proceedings of the 2013 IEEE Grenoble PowerTech Conference, Grenoble, France, 16-20 June 2013. 
36. McNabb, P.; Bialek, J.W. A priori transient stability indicator of islanded power systems using extended equal area criterion. In Proceedings of the IEEE Power Engineering Society General Meeting, San Diego, CA, USA, 22-26 July 2012.

37. Xue, Y.S.; Ribbens-Pavella, M. Critical cluster identification in transient studies (of power systems). IEE Proc. C Gener. Trans. Distrib. 1993, 140, 481-489. [CrossRef]

38. Norris, S. Preventing Wide Area Blackouts in Transmission Systems: A New Approach of Controlled Islanding Using Power Flow Tracing. Ph.D. Thesis, Durham University, Durham, UK, 2014.

39. Pai, M.A. Energy Function Analysis for Power System Stability; Kluwer Academic Publishers: Boston, MA, USA, 1989.

(C)

(C) 2018 by the authors. Licensee MDPI, Basel, Switzerland. This article is an open access article distributed under the terms and conditions of the Creative Commons Attribution (CC BY) license (http://creativecommons.org/licenses/by/4.0/). 